\title{
Vps1 in the late endosome-to-vacuole traffic
}

J Hayden

MSU Graduate Student

M Williams

MSU Undergraduate

A Granich

MSU Undergraduate

H Ahn

MSU Undergraduate

B Tenay

MSU Graduate Student

See next page for additional authors

Follow this and additional works at: https://bearworks.missouristate.edu/articles-cnas

\section{Recommended Citation}

Hayden, Jacob, Michelle Williams, Ann Granich, Hyoeun Ahn, Brandon Tenay, Joshua Lukehart, Chad Highfill, Sarah Dobard, and Kyoungtae Kim. "Vps1 in the late endosome-to-vacuole traffic." Journal of biosciences 38, no. 1 (2013): 73-83.

This article or document was made available through BearWorks, the institutional repository of Missouri State University. The work contained in it may be protected by copyright and require permission of the copyright holder for reuse or redistribution.

For more information, please contact BearWorks@library.missouristate.edu. 


\section{Authors}

J Hayden, M Williams, A Granich, H Ahn, B Tenay, J. Lukehart, C Highfill, S Dobard, and Kyoungtae Kim 


\title{
Vps1 in the late endosome-to-vacuole traffic
}

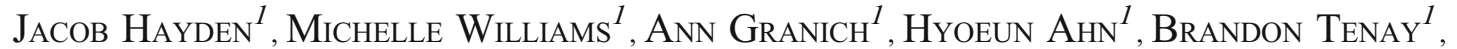 \\ Joshua Lukehart ${ }^{l}$, Chad Highfill $^{1,2}$, SArah Dobard ${ }^{l}$ and Kyoungtae Kim ${ }^{1, *}$ \\ ${ }^{1}$ Department of Biology, Missouri State University, 901 S National, Springfield, Missouri 65807 \\ ${ }^{2}$ Department of Molecular Biosciences, University of Kansas, 1200 Sunnyside Avenue, \\ Lawrence, Kansas 66045
}

*Corresponding author (Fax, +1-417-8365126; Email, kkim@missouristate.edu)

\begin{abstract}
Vacuolar protein sorting 1 (Vps1), the yeast homolog to human dynamin, is a GTP hydrolyzing protein, which plays an important role in protein sorting and targeting between the Golgi and late endosomal compartments. In this study, we assessed the functional significance of Vps1 in the membrane traffic towards the vacuole. We show here that vps $1 \Delta$ cells accumulated FM4-64 to a greater extent than wild-type (WT) cells, suggesting slower endocytic degradation traffic toward the vacuole. In addition, we observed that two endosome-to-vacuole traffic markers, DsRed-FYVE and Ste2-GFP, were highly accumulated in Vps1-deficient cells, further supporting Vps1's implication in efficient trafficking of endocytosed materials to the vacuole. Noteworthy, a simultaneous imaging analysis in conjunction with FM4-64 pulse-chase experiment further revealed that Vps1 plays a role in late endosome to the vacuole transport. Consistently, our subcellular localization analysis showed that Vps1 is present at the late endosome. The hyperaccumulation of endosomal intermediates in the vps 1 mutant cells appears to be caused by the disruption of integrity of HOPS tethering complexes, manifested by mislocalization of Vps39 to the cytoplasm. Finally, we postulate that Vps1 functions together with the Endosomal Sorting Complex Required for Transport (ESCRT) complex at the late endosomal compartments, based on the observation that the double mutants, in which VPS1 along with singular ESCRT I, II and III genes have been disrupted, exhibited synthetic lethality. Together, we propose that Vps1 is required for correct and efficient trafficking from the late endosomal compartments to the vacuole.
\end{abstract}

[Hayden J, Williams M, Granich A, Ahn H, Tenay B, Lukehart J, Highfill C, Dobard S and Kim K 2013 Vps1 in the late endosome-to-vacuole traffic. J. Biosci. 38 73-83] DOI 10.1007/s12038-012-9295-2

\section{Introduction}

Vacuolar protein sorting 1 (Vps1), first discovered by Rothman and Stevens in 1986 (Rothman and Stevens 1986), encodes an $80 \mathrm{kDa}$ GTPase that is considered to be a yeast homolog of the mammalian dynamin (Conibear and Stevens 1995). Dynamin is a multi-domain GTPase protein that has been shown to be involved in endocytic processes at the plasma membrane (Wiejak and Wyroba 2002). Dynamin has also been implicated in vesicle formation at the trans-Golgi network (Praefcke and McMahon 2004). Vps1 and dynamin share $45 \%$ similarity in their sequences and also have similar structures (Obar et al. 1990). Dynamin is composed of five functionally different domains: the GTPase domain, the middle domain, the pleckstrin homology domain $(\mathrm{PH})$, the
GTPase effector domain (GED) and the C-terminal proline-rich domain (PRD) (Ramachandran 2011). However, Vps1 consist of only the N-terminal GTPase domain, the middle domain and the GED domain (Vater et al. 1992; Yu and Cai 2004).

Vps1 has been implicated in several intracellular membrane fission events at the vacuole, Golgi and peroxisomal systems (Hoepfner et al. 2001; Nothwehr et al. 1995; Peters et al. 2004). Vps1's vacuolar protein sorting function was first discovered while screening for yeast mutants that failed to properly deliver carboxypeptidase Y (CPY) and proteinase A to the vacuole (Rothman and Stevens 1986). CPY is synthesized in the endoplasmic reticulum (ER) and normally delivered to the vacuole via the Golgi and late endosome/ prevacuolar compartment (Conibear and Stevens 1995).

Keywords. ESCRT; FM4-64; HOPS; late endosome; traffic; vacuole; Vps1 
However, without Vps1, CPY is diverted to the outside of the cell via an intact secretory pathway (Rothman and Stevens 1986). In addition, cells deficient of Vps1 missort Golgi membrane proteins, such as Kex2, which normally shuttles between the late endosome/PVC and the late Golgi. In $v p s 1 \Delta$ cells, Kex 2 proteins are rerouted to the plasma membrane where they reach the vacuole only after being endocytosed (Nothwehr et al. 1995). This process is only feasible if the secretory protein, Sec4, and the endocytic protein, End4, are present (Nothwehr et al. 1995).

Due to the fact that an earlier yeast mutation screening analysis failed to find the significance of Vps1 in endocytosis and that Kex2 was found at the vacuolar membrane in vps 1 mutant cells, it has been assumed that endocytosis and the following post-endocytic traffic toward the vacuole in the mutants are not affected. However, with the recent advancement in live-cell imaging of endocytic events using fluorescence microscopy technology, groups of researchers have shown that Vps1 plays an important role in endocytosis. First, Yu and Cai (2004) showed that loss of Vps1 resulted in a reduced efficiency in the internalization of the membrane receptor Ste3. Second, vps $1 \Delta$ cells often exhibited an aberrant shape of endocytic invaginations, indicating that Vps1 functions in facilitating membrane invagination, which may lead to an effective scission of the vesicle from the plasma membrane (Smaczynska-de et al. 2010). Third, Vps1 was found to genetically and physically interact with an amphiphysin, Rvs167; it was suggested that the interaction between Vps1 with Rvs167 is necessary for vesicle scission during endocytosis (Smaczynska-de et al. 2010; Smaczynska-de et al. 2012). Fourth, our lab reported that the loss of Vps1 triggers the formation of aberrant actin cables that affects endocytic vesicle internalization motility (Nannapaneni et al. 2010).

We have begun an analysis of Vps1 using confocal fluorescence microscopy equipped with a simultaneous dualimaging technology in an attempt to gain insight into its role in the post-endocytic vesicle traffic destined for the vacuole. We for the first time provide evidence that Vps1 plays a role in the traffic from the late endosomal compartment to the vacuole. We also demonstrate that VPS1genetically interacts with the genes encoding protein components of endosomal ESCRT complexes. Together, these findings reveal a new role for Vps1 in the endocytic traffic toward the vacuole.

\section{Materials and methods}

\subsection{Strain construction and media}

Strains expressing Ste2-GFP were constructed by introducing the plasmid pRS314STE2-GFP (Stefan and Blumer 1999) by following one-step transformation protocol (Chen et al. 1992). The plasmid encoding DsRed-FYVE (a kind gift from Dr. Nava Segev) was introduced to construct strains that express DsRed-FYVE, marking endosomes. GFP-Pep12 expressing strains were constructed by integrating pGAL-GFP construct at the $5^{\prime}$ end of the PEP12 coding region by a PCR-based gene modification, as previously described (Longtine et al. 1998). Strains expressing Vps3GFP, Vps8-GFP, Vps39-GFP, Vps41-GFP, Vps1-GFP and Vps1-RFP were constructed by integrating GFP (or RFP) construct at the $3^{\prime}$ end of the corresponding coding region, as previously described (Longtine et al. 1998). Yeast strains were grown in standard yeast peptone dextrose (YPD) medium unless otherwise stated. Cells expressing DsRedFYVE were grown in SD-LEU media. GFP-Pep12 expressing cells were cultured in SD-HIS-containing raffinosecontaining media, and raffinose was replaced by galactose to induce expression of GFP-Pep12. Double mutant cells used in this study were generated by crossing single-genedeficient strains, followed by tetrad dissection.

\subsection{FM4-64 labelling}

Cells were incubated with $25 \mu \mathrm{M}$ FM4-64 (Molecular Probes Inc., Eugene, OR) on ice for $5 \mathrm{~min}$ and then washed with ice-cold SD media. The cells were then incubated for various times (up to $60 \mathrm{~min}$ ) with $\mathrm{SD}$ media at $30^{\circ} \mathrm{C}$ prior to microscopic analysis.

\subsection{CMAC labeling of yeast vacuole membranes}

CMAC staining of vacuoles was done by incubating cells for 15 min at $30^{\circ} \mathrm{C}$ with CellTracker Blue ${ }^{\mathrm{TM}} \mathrm{CMAC}$ (7-amino4 chloromethylcoumarin, Molecular probes Inc.), followed by washing with YPD media and another $30 \mathrm{~min}$ incubation. The stained cells were then subjected to microscopic analysis.

\subsection{Confocal fluorescence microscopy}

Digital images of GFP-, RFP-, FM4-64, CMAC-labelled cells were made with a spinning confocal system that includes an upright Olympus IX81 microscope, a Yokogawa CSUX1 spinning disk head, a 100X numerical aperture (NA) 1.4 Plan Apo oil objective and an electron Amplified CCD (ImagEM, Hamamatsu). In all cases, the laser beams were focused at the equatorial plane of the cells. Unless otherwise stated, the temperature of the specimen and stage was maintained at $30^{\circ} \mathrm{C}$.

\subsection{Spotting yeast cells for cell growth assay}

We performed spotting assays on YPD plates to quantitatively assess the impact of double mutations on cell growth. 
To do so, cells were diluted by a factor of 5 and grown for 2 days at $30^{\circ} \mathrm{C}$ and $37^{\circ} \mathrm{C}$.

\section{Results and Discussion}

\subsection{Loss of Vps1 affects trafficking in the endosome-to- vacuole}

It has been proposed that loss of function of Vps1 leads to blocking of membrane traffic from the late Golgi to the prevacuolar compartment (late endosome) (Nothwehr et al. 1995). Therefore, it appears that the Golgi resident protein Kex2, normally shuttling between the late Golgi and endosomes, is first delivered to the plasma membrane in vps 1 mutant cells and then to the vacuole via endocytosis. It was proposed that the latter pathway is dependent on the presence of intact End4 (Nothwehr et al. 1995). Recent findings with fluorescence microscopy indeed revealed that endocytosis occurs in vps $1 \Delta$ cells, albeit incompetently (Nannapaneni et al. 2010; Smaczynska-de et al. 2010, 2012). These findings inspired us to assess Vps1's role in the post-endocytic vesicle traffic destined for the vacuole, using well-known markers that follow the traffic. FM4-64 is a dye that binds to the plasma membrane lipids and follows the endocytic internalization pathways (Vida and Emr 1995). Post-internalized endocytic vesicles that carry FM4-64 fuse with early endosomes. The majority of the dye recycles back to the plasma membrane and to the culture media in $10 \mathrm{~min}$, directly from the early endosomes and/or travelling to the Golgi and secreted out by secretory vesicles (Wiederkehr et al. 2000). The rest of the FM4-64, from the early endosome, follows the endocytic degradation pathway toward the vacuole (Vida and Emr 1995). Here, our pulse-chase experiments using FM4-64 revealed that the dye was found exclusively at the rim of the vacuole in wild-type (WT) cells after a 30 min chase, while in $v p s 1 \Delta$ cells, the FM4-64 was mainly dissipated in the cytoplasm and found also as punctuate structures (figure 1A). Our explanation for the cytoplasmic hyperaccumulation of FM4-64 in vps $1 \Delta$ cells is that at least endosome-to-vacuole traffic is impaired, suggesting the role of Vps1 in the efficient trafficking of the endocytosed membrane toward the vacuole. Furthermore, our data indicates that recycling traffics of FM4-64 is also attenuated in the vps 1 mutant cells.

Yeast endosomal membranes contain PI(3)P that binds to proteins containing FYVE finger domain (Burd and Emr 1998; Stenmark and Aasland 1999). In light of the finding of Vps1's implication in endocytic trafficking toward the vacuole, we investigated whether the loss of Vps1 affects the integrity of $\mathrm{PI}(3) \mathrm{P}$-positive endosome. To this end, we expressed the plasmid encoding DsRed-FYVE by constructing strains that express DsRed-FYVE, marking endosomes. In WT cells (figure 1B) only a few (often one or two) endosomes marked with DsRed-FYVE were seen as punctuate structures adjacent to the vacuole. However, in vps $1 \Delta$ cells, many endosomes labelled with DsRed-FYVE reporter were seen as clustered or aggregated structures at or near the vacuole (figure 1B). Given that the PI(3)P-positive endosome represents endocytic intermediate (Gillooly et al. 2000), most likely representing late endosomal compartments, our PI(3)P-endosome accumulation data serves as an another solid evidence that supports the function of Vps1 in the traffic of endosome toward the vacuole.

Ste2 is a G-protein-coupled receptor that follows endocytic traffic destined for the vacuole (Schandel and Jenness 1994). It is known that Ste 2 is constitutively endocytosed at a relatively slow rate and is degraded in the lysosome-like vacuole in the absence of its ligand, the yeast mating pheromone $\alpha$-factor (Hicke and Riezman 1996; Hicke et al. 1997, 1998). Based on the results obtained from experiments using FM4-64 and the endosome marker DsRed-FYVE, we reasoned that the endocytic trafficking of Ste 2 could be affected in $v p s 1 \Delta$ cells. To test the possibility, we expressed pRS314STE2-GFP plasmid (Stefan and Blumer 1999) in yeast strains and examined the localization of Ste2-GFP. Consistent with the data published previously (Chang et al. 2003; Stefan and Blumer 1999), WT cells were observed to have membrane Ste2-GFP staining along with approximately two Ste2-GFP puncta (figure 1C-D) in the cytoplasm, as well as with some vacuolar lumen staining. Ste2-GFP fluorescence in $v p s l \Delta$ cells, as shown in figure $1 \mathrm{C}$, often led to hazy staining of the cytoplasm, and the mutant cells exhibited an increase in Ste2-GFP puncta number (average of 6.9) (figure 1D). The abnormal distribution and the increased puncta number of Ste2-GFP in the mutant may not be caused by a secretory defect due to the fact that integrated membrane proteins such as Pmal and Sncl are properly targeted to the plasma membrane (Luo and Chang 2000) (unpublished results from our Lab). Instead, our current best explanation for the Ste2-GFP accumulation in the cytoplasm in vps 1 mutant cells (figure 1C) would be due to a defect in the endocytic trafficking en route to the vacuole.

In addition to the Ste2-GFP localization assay in the absence of $\alpha$-factor, we performed an experiment in which Ste2GFP endocytosis was stimulated by exposing cells to $\alpha$-factor $(0.5 \mu \mathrm{M})$ over a $6 \mathrm{~h}$ period. As expected, the average number of Ste2-GFP puncta that do not overlap with CMAC-stained vacuole drastically increased over time in Wt cells, peaked at $2 \mathrm{~h}$ after $\alpha$-factor treatment, with the average of 9.8 in Ste2GFP puncta number (figure 1D). After $3 \mathrm{~h}$ of $\alpha$-factor treatment in Wt cells, we observed a twofold reduction $(\sim 4.8)$ in the average number of Ste2-GFP puncta, when compared to that of $2 \mathrm{~h}$ treatment. This result suggests that, after $2 \mathrm{~h}$ of incubation with $\alpha$-factor, the endocytosed Ste2-GFP proteins are destined for the vacuolar lumen, manifested by an intense Ste2-GFP fluorescence in the vacuolar lumen in WT cells (figure 1D). However, in the vps $1 \Delta$ mutant cells, the average 
(A)

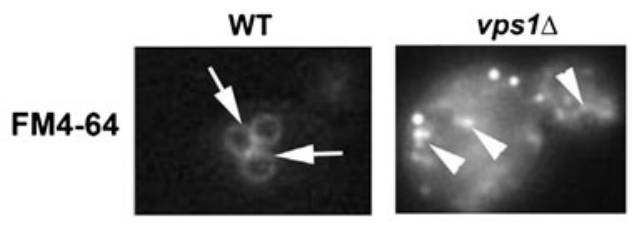

(B)

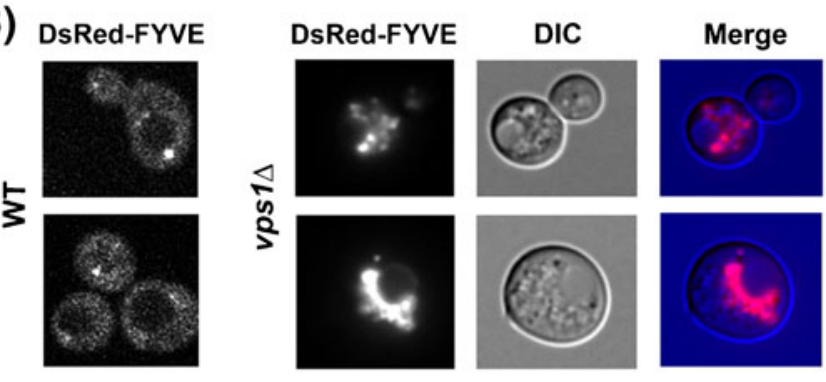

(C)

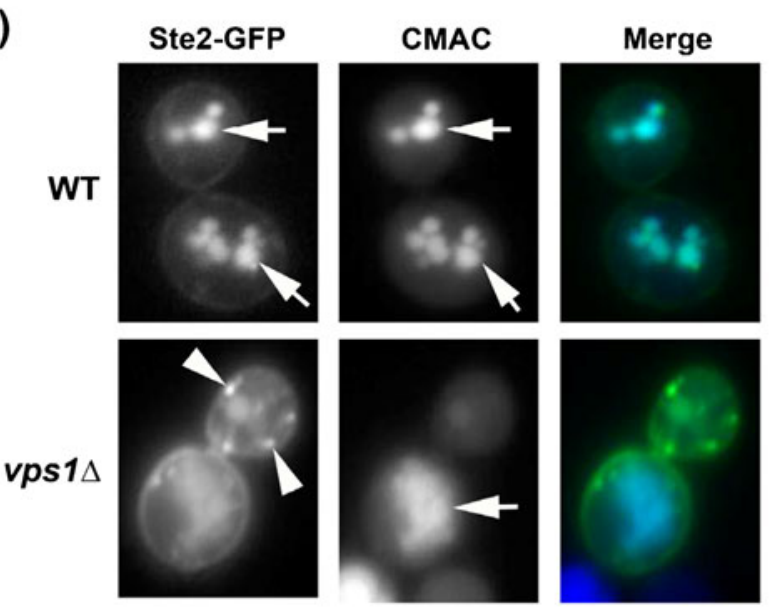

(D)

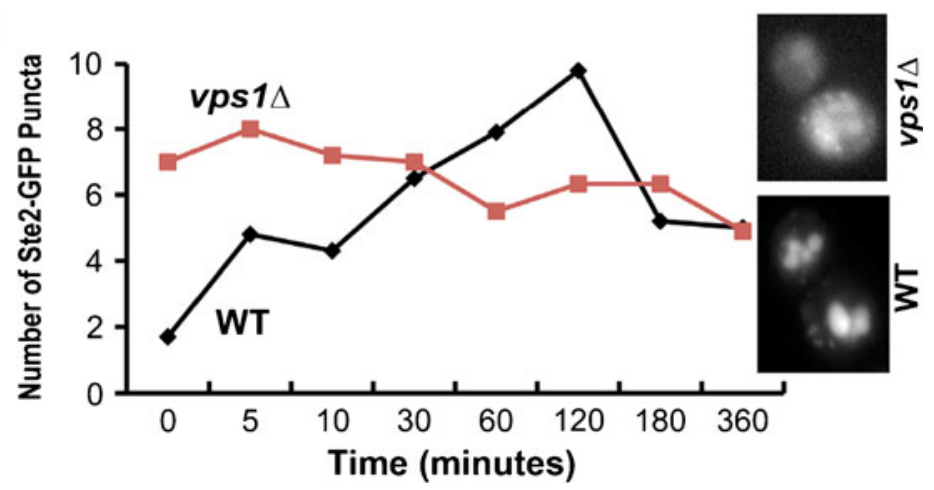

Figure 1. Loss of Vps1 results in traffic defect toward the vacuole. (A) Cytoplasmic accumulation FM4-64. WT (KKY 343) and vps1 $\Delta$ (KKY 352) cells were incubated with $12 \mu \mathrm{L}$ of $1 \mathrm{mM} \mathrm{FM} 4-64$ for $5 \mathrm{~min}$ and chased for $30 \mathrm{~min}$ at $30^{\circ} \mathrm{C}$. The rim of the vacuole stained with FM4-64 in a WT cell is indicated by arrows. vps $1 \Delta$ cells show cytoplasmic haze and punctate structures of FM4-64 (arrowheads). (B) Accumulation of endosomes. WT (KKY 002) cells expressing DsRed-FYVE were visualized using an Olympus IX81 inverted spinning confocal microscope equipped with ImagEM camera. WT cells show clear puncta in the cytoplasm marking the endosomes, whereas $v p s 1 \Delta$ cells expressing DsRed-FYVE show an accumulation of endosomal compartments. (C) Ste2-GFP accumulation. Ste2-GFP-expressing cells were stained with CMAC. In WT cells, one or two small Ste2-GFP patches that do not overlap with CMAC are often observed. In addition to forming a dense cytoplasmic haze, Ste2-GFP appeared as punctate structure in $v p s 1 \Delta$ cells. White arrows indicate vacuoles, while arrowheads highlight Ste2-GFP carrying endosomes. (D) Ste2-GFP in the presence of $\alpha$-factor. Over $6 \mathrm{~h}$ of period with $\alpha$-factor, the average number of Ste2-GFP puncta in WT and vps $1 \Delta$ cells was determined. 
number $(\sim 6)$ of Ste2-GFP puncta at $2 \mathrm{~h}$ of incubation did not essentially change when compared to that at time 0 (6.9). After $2 \mathrm{~h}$ with $\alpha$-factor, it appeared that the Ste2-GFP puncta number decreased in the mutant cells, albeit not significantly. Our explanation for no significant change in Ste2-GFP puncta number during the entire timecourse in the mutant cells would be that loss of Vps1 may lead both to an inefficient internalization of Ste2 via receptor-mediated endocytosis during the first $2 \mathrm{~h}$ and to a significantly slow traffic of Ste2 toward the vacuole for its degradation.

Together, our assessment regarding the role of Vps1 in post-endocytic traffic reinforces our proposed role of Vps1 in the proper trafficking of endocytosed materials to the vacuole, which might have been overlooked over the years. Therefore, it is most likely that the delivery of the endocytosed molecules including Ste2 and Golgi resident proteins (Kex2 and Vps10) in cells deficient of Vps1 is severely affected, due to an extremely inefficient traffic bound for the vacuole.

\subsection{Traffic from late endosome to the vacuole is impaired in vps 1 mutant cells}

Once extracellular material has been internalized by endocytosis, it moves through two membrane-bound compartments, characterized as early and late endosomes (Munn 2000; Singer-Kruger et al. 1993; Singer and Riezman 1990). The contents from the late endosome are then transferred to the vacuole and degraded (Munn 2000; SingerKruger et al. 1993; Singer and Riezman 1990). Transport between endosomes and the vacuole requires many components such as Rab-family GTPases, v-SNAREs and t-SNAREs (Munn 2000). To determine whether Vps1 is required for the traffic toward the late endosome or the traffic from the late endosome to the vacuole, cells were made to express GFP-Pep12 and subjected to a FM4-64 chase experiment. Pep12 is a syntaxin-like t-SNARE that is found to be located primarily at the late endosome or pre-vacuole compartment (PVC), distinct from the Golgi and vacuolar compartments (Becherer et al. 1996). After $10 \mathrm{~min}$ chase, the FM4-64 dye both in WT and vps $1 \Delta$ cells was seen to colocalize with GFP-Pep12, indicating that the FM4-64 has made it to the late endosome/PVC compartment (figure 2A). Noteworthy, we observed that vps $1 \Delta$ cells exhibited many more GFP-Pep12 puncta than WT cells did and higher GFP-Pep12 fluorescence intensity puncta, suggesting higher accumulation of Pep12 (figure 2A). Although vps 1 mutant cells slow down the pinch-off process to form a membrane-bound endosome, as has been shown by our group and others (Nannapaneni et al. 2010; Smaczynska-de et al. 2010; Smaczynska-de et al. 2012), based on our result here we can conclude that traffic toward the late endosome can be completed within $10 \mathrm{~min}$ in vps $1 \Delta$ cells. Thus, it is likely that the earlier endocytic invagination/internalization defect in $v p s 1 \Delta$ cells yields negligible impact on the accumulation of endocytic intermediates (figure 1). By 60 minute, wild-type cells showed a vacuole ring stained with FM4-64, whereas in vps $1 \Delta$ cells exhibited no vacuole ring stained with FM464. Instead, the FM4-64 dye was mainly colocalized with GFP-Pep12 in the mutant cells, indicating that the FM4-64 dye is not being properly trafficked to the vacuole, from the late endosome/PVC (figure 2B). Overall, our results suggest that $\mathrm{Vps} 1$ 's role for the proper trafficking from the late endosome/PVC to the vacuole is more important than its role for the traffic toward the late endosome.

\subsection{Vps1 is localized to late endosomal compartments}

Given that subcellular localization of a protein is closely correlated with its biological functions, we examined whether or not Vps1 is localized to endosomal compartments. To assess whether Vps1 is at the early or later endosomal systems, cells expressing Vps1-GFP were pulsed with FM4-64 for 10 min on ice and chased for up to $20 \mathrm{~min}$ at $30^{\circ} \mathrm{C}$. Our results revealed that after 1 min chase, Vps1-GFP showed very little colocalization (less than 5\%) with FM4-64 (figure 3A-B). By 5 min, about $22 \%$ of FM4-64 puncta showed colocalization with Vps1-GFP patches. The extent of colocalization between them reached $50 \%$ after $20 \mathrm{~min}$ chase (figure $3 \mathrm{~A}-\mathrm{B}$ ). Since FM4-64 transit to late endosomal compartments can be done in $10 \mathrm{~min}$ as shown earlier (figure 2A), higher extent of colocalization between Vps1-GFP and FM4-64, after $20 \mathrm{~min}$ chase, indicates that Vps1-carrying vesicles are among the population of late endosomal/PVC compartments. DsRedFYVE has been found to specifically bind to PI(3)P-enriched endosomal membranes (Burd and Emr 1998). Importantly, Gillooly et al. (2000) showed that DsRed-FYVE in yeast is localized by electron microscopy to internal vesicles near and evidently within the vacuole, thus representing bona fide late endosomes. Accordingly, we examined the possible colocalization of Vps1-GFP with DsRed-FYVE. When marked with DsRed-FYVE, endosomes appear as punctuate structures, near the vacuole (figure 3C). When Vps1-GFP was expressed in cells harbouring DsRed-FYVE, cytoplasmic Vps1-GFP puncta were seen to colocalize with DsRed-FYVE (figure 3C), confirming that Vps1 is present among the late endosomal system. Finally, we checked whether Vps1-RFP colocalizes with GFP-Pep12, a late endosome marker. As shown, higher levels of colocalization of Vps1-RFP with GFP-Pep12 (figure 3D) further support that the primary localization of $\mathrm{Vps} 1$ is at the late endosome/PVC.

\subsection{Functional interaction between Vps1 and ESCRT complex}

Ste2 is a surface receptor that is endocytosed in a ubiquitindependent manner (Hicke and Riezman 1996). The 

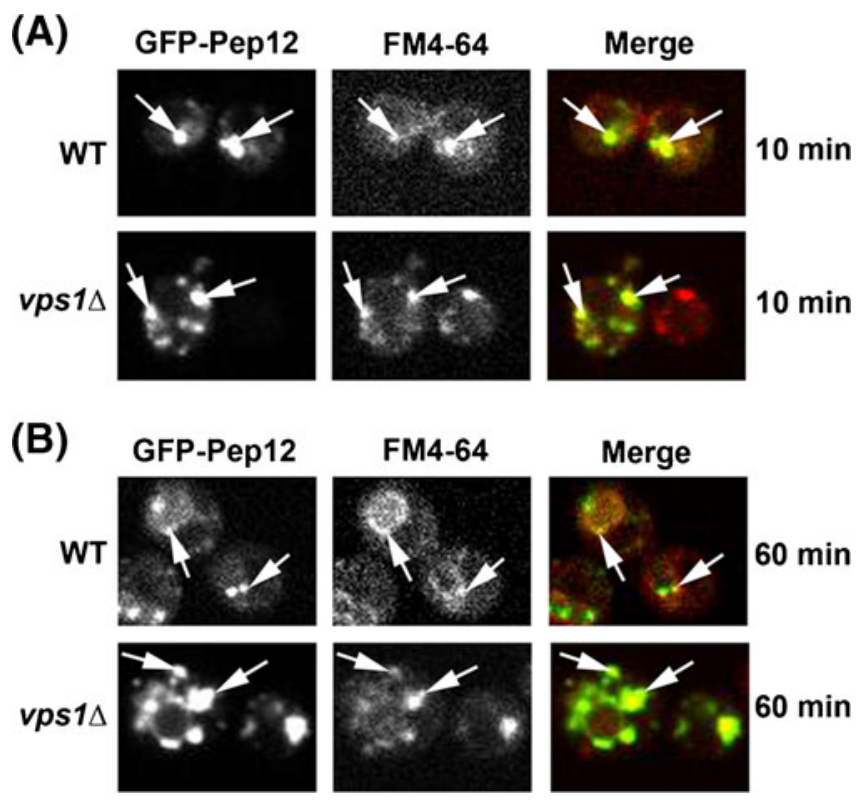

Figure 2. Vps1 functions in the late endosome to vacuole transport. (A) Subcellular localization of FM4-64, after 10 min chase. WT cells expressing GFP-Pep12 (KKY 334) were incubated with $12 \mu \mathrm{L}$ of $1 \mathrm{mM}$ FM4-64 and chased for 10 min at $30^{\circ} \mathrm{C}$. Arrows show colocalization between GFP-Pep12 and FM4-64 at 10 min in WT cells (upper panels). vps $1 \Delta$ cells expressing GFP-Pep12 (KKY 335) also show colocalization between FM4-64 and GFP-Pep12 (arrows, lower panels). (B) Subcellular localization of FM4-64, after 60 min chase. In WT cells, FM4-64 was visualized at the rim of vacuole and a few FM4-64 puncta colocalized with GFP-Pep12 (arrows, upper panels), showing proper transport of FM4-64 dye to the vacuole. However, in vps $1 \Delta$ there appears to be no vacuolar staining; FM4-64 instead colocalizes with the GFP-Pep12 puncta (arrows, bottom panels).

ubiquitinated Ste2 proteins are then delivered to the late endosome/multivesicular body (MVB) compartment in which they are sorted for delivery to the intralumenal vesicles (ILV) for final degradation in the vacuole (Stefan and Blumer 1999). Based on the result that Ste2-cargo sorting on endosomal membrane require the activity of ESCRT (endosomal sorting complex required for transport) (Morvan et al. 2012), we reasoned that the slow Ste2-GFP traffic toward the vacuole in Vps1-deficient cells was probably due to a disruption of ESCRT integrity. Our approach to define the functional relationship between Vps1 and subunits of ESCRT was producing combination mutants in two genes to observe genetic interactions. Mutations of two nonessential genes could lead a lethal phenotype, known as synthetic lethality interaction (SLI). SLI suggests the genes or their products are required for redundant biological processes or function in parallel pathways (Tucker and Fields 2003), without any requirement that proteins must directly interact. In order to find potential ESCRT genes that interacts with $V P S 1$, we generated 1 haploid mutant of ESCRT 0

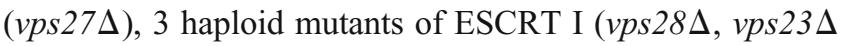

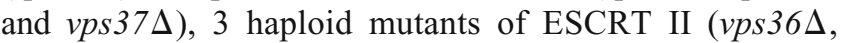
$v p s 25 \Delta$ and vps $22 \Delta$ ), and 3 mutants of ESCRT III (vps $24 \Delta$, vps $32 \Delta$ and vps $2 \Delta$ ). All single-knockout mutants of ESCRT and Vps1 were viable, as expected, both at $30^{\circ} \mathrm{C}$ and $37^{\circ} \mathrm{C}$, but $v p s 28 \Delta$, vps $25 \Delta$ and $v p s 22 \Delta$ cells exhibited slow growth in a growth assay (figure 4A). Next, each ESCRT haploid mutant was crossed with vps $1 \Delta$ to produce a heterozygous diploid, which was then subjected to sporulation and tetrad dissection. It was observed that all double mutant cells grow slowly except only three double mutants (vps $1 \Delta v p s 27 \Delta$, vps $1 \Delta v p s 24 \Delta$ and vps $1 \Delta v p s 2 \Delta$ ) that showed normal growth at $30^{\circ} \mathrm{C}$. Strikingly, 7 double mutants (vps $1 \Delta v p s 28 \Delta, v p s 1 \Delta v p s 3 \Delta, v p s 1 \Delta v p s 37 \Delta$, vps $1 \Delta v p s 36 \Delta$, vps $1 \Delta v p s 25 \Delta$, vps $1 \Delta v p s 22 \Delta$ and vps $1 \Delta v p s 24 \Delta$ ) were inviable at $37^{\circ} \mathrm{C}$ (figure $4 \mathrm{~B}$ ). Therefore, our results indicate the functional interaction between Vps1 and the ESCRT complex, in particular, ESCRT I (Vps28, Vps23 and Vps37), ESCRT II (Vps36, Vps25 and Vps22), and ESCRT III (Vps24).

\subsection{Vps1 affects the localization of subunits of HOPS and CORVET tethering complexes}

Endosomal transport occurs from the maturation of early endosomes into late endosomes and, in particular, late endosomes form intralumenal vesicles and the resulting multivesicular bodies (MVBs) fuse with the vacuole to release their cargo (Spang 2009). The fusion event at the vacuole is first mediated by the vacuolar HOPS (homotypic fusion and vacuole protein sorting) tethering complex (Nickerson et al. 2009; 

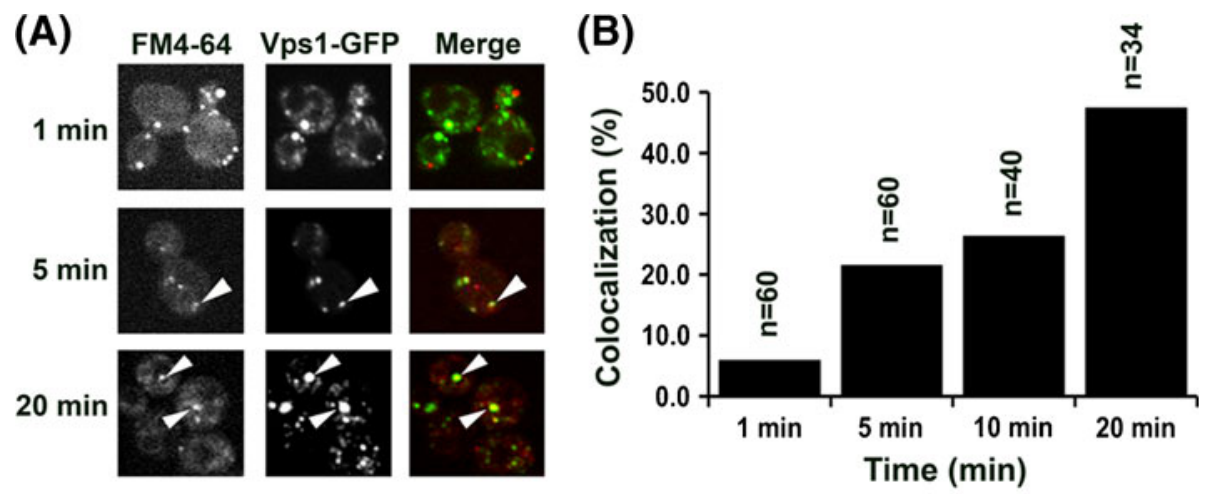

(C)

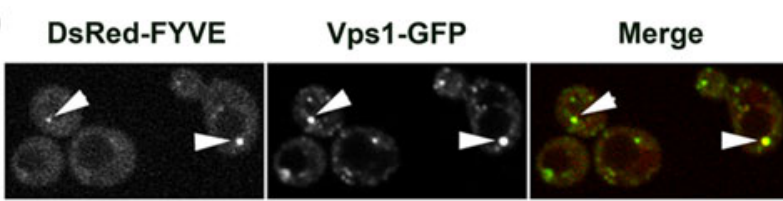

(D)
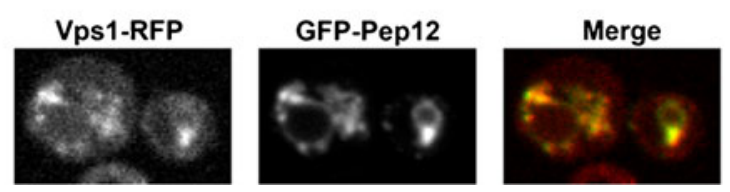

Figure 3. Vps1 is mainly colocalized to late endosomes. (A) Colocalization between Vps1-GFP and pulse-chased FM4-64. Arrowheads indicate colocalization between FM4-64 and Vps1-GFP. (B) The extent of colocalization of FM4-64 with Vps1-GFP. The extent of colocalization between FM4-64 and Vps1-GFP increased over the 20 min FM4-64 chase, indicating that Vps1 is primarily located at the late endosome/PVC. (C) Vps1-GFP colocalizes with DsRed-FYVE, an endosomal marker. The arrowheads show points of colocalization between DsRed and Vps1-GFP. (D) Colocalization of Vps1-RFP with GFP-Pep12.

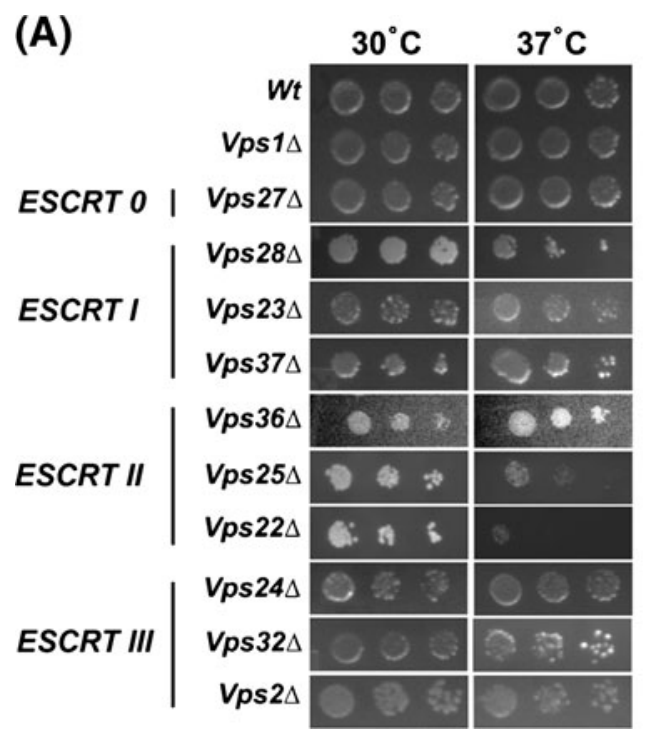

(B)

\begin{tabular}{|c|c|c|}
\hline & $30^{\circ} \mathrm{C}$ & $37^{\circ} \mathrm{C}$ \\
\hline$V p s 1 \Delta V p s 27 \Delta$ & 000 & 00 \\
\hline Vps1 $1 \Delta p s 28 \Delta$ & $0 *$ & \\
\hline$V p s 1 \Delta V p s 23 \Delta$ & $028:$ & \\
\hline$V p s 1 \Delta V p s 37 \Delta$ & $0: 5$ & 0 \\
\hline$V p s 1 \Delta V p s 36 \Delta$ & $00=$ & \\
\hline$V p s 1 \Delta V p s 25 \Delta$ & 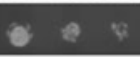 & \\
\hline$V p s 1 \Delta V p s 22 \Delta$ & a 5 & \\
\hline$V p s 1 \Delta V p s 24 \Delta$ & 008 & \\
\hline$V p s 1 \Delta V p s 32 \Delta$ & 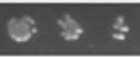 & 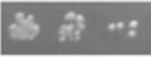 \\
\hline$V p s 1 \Delta V p s 2 \Delta$ & 008 & 003 \\
\hline
\end{tabular}

Figure 4. Genetic interactions between vps 1 mutant and ESCRT mutants. (A) Growth assay using single-knockout strains. Heterozygous diploids were generated by crossing vps $1 \Delta$ cells with single ESCRT gene knockout strains. After dissecting tetrads from the heterozygous diploids, each single knockout strain was grown at $30^{\circ} \mathrm{C}$ and $37^{\circ} \mathrm{C}$ by spotting fivefold diluted cultures onto YPD plates. (B) Growth assay using double-knockout strains. The indicated haploid double-knockout strains were grown in the same way as described above. 
Ostrowicz et al. 2008). The HOPS complex consists of four class C core proteins, Vps11, Vps16, Vps18 and Vps33, along with two additional subunits, Vps39 and Vps41 (Brett et al. 2008; Price et al. 2000; Wurmser et al. 2000). The endosomal tethering complex, CORVET (class C core vacuole/ endosome tethering), functions between the early and late endosome/PVC and shares the same class $\mathrm{C}$ core proteins as the HOPS complex and two specific subunits, Vps3 and Vps8 (Peplowska et al. 2007). Based on the observations of the accumulation of FM4-64 in late endosomes/PVC upon the deletion of VPS1, we tested the possibility that the localization of HOPS subunits may be disturbed in vps $1 \Delta$ cells. Consistent with the results of Cabrera et al (2010), our results showed that HOPS subunits, Vps39- and Vps41GFP, are localized to the vacuole (figure $5 \mathrm{~A}$ ) and vacuoleassociated patch-like structure (possibly late endosomes/ PVC) in wild-type cells. However, in vps1 $1 \Delta$ cells Vps39GFP, but not Vps41-GFP, was dispersed in the cytoplasm $(\sim 80 \%$ of vps 1 mutant cells), with increased cytoplasmic GFP intensity (figure 5A). Given that those vacuolar proteins play a critical role in the tethering steps of endosome-vacuole fusion (Cabrera et al. 2010; Wurmser et al. 2000), our results suggest the possibility that Vps1 functions in proper recruitment of Vps39, a HOPS subunit, to the vacuolar membrane. Upon the observation of Vps39 mislocalization, we asked whether loss of Vps1 affects the integrity of CORVET tethering complex at late endosomal compartments. We speculate that this would be the case since an earlier study showed that Golgi-to-late endosome transport is blocked in vps 1 mutant cells (Nothwehr et al. 1995). As stated earlier, in the endosomal tethering complex CORVET, Vps39 and Vps41 are replaced by the homologous proteins Vps3 and Vps8 (Markgraf et al. 2009), and this interconversion between subunits occurs during endosome-vacuole transition (Peplowska et al. 2007). To investigate whether

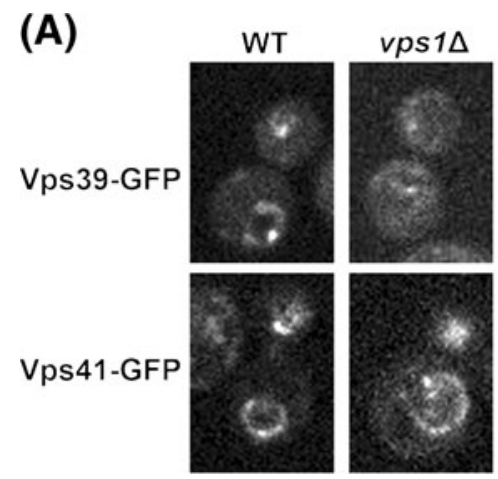

loss of Vps1 affects the recruitment of Vps3 and Vps8 to endosomal compartments, we examined the localization of $\mathrm{Vps} 3$ - and Vps8-GFP. As shown in figure 5B, in $v p s 1 \Delta$ cells, the GFPfusion proteins were more dispersed with an increased cytoplasmic staining. Together, our results suggest that Vps1 may play a role in the recruitment of protein subunits that are essential for the CORVET and HOPS complexes.

In our present study, several lines of evidence point to a hitherto unrecognized function of Vps1 in endocytic traffic toward the vacuole, in particular trafficking from the late endosome to the vacuole. Traditionally, VPS1 has been classified into the class F VPS gene (Raymond et al. 1992), and vps 1 mutant cells contain a large central vacuole surrounded by a number of fragmented vacuoles. Interestingly, according to our result here, the aberrant phenotype of $v p s 1 \Delta$ cells exhibiting the accumulation of $\mathrm{PI}(3) \mathrm{P}$-positive endosomes near the vacuole is very reminiscent of that of $v p s$ class E mutant cells. More importantly, most of previously characterized 13 class E VPS genes (Vps2, 4, 20, 22, $23,24,25,27,28,31,32,36$ and 37) that encode proteins forming ESCRT complex (0, I, II and III) show synthetic lethality interaction with VPS1. These results support a model wherein Vps1, predominantly present on late endosomes (figures 3 and 6), may function together or redundantly with ESCRT complex in protein sorting and targeting at late endosomes (figures 4 and 6). We further concluded that Vps1 is required for proper recruitment of the tethering factor Vps39 on the vacuole (figures 5 and 6), which facilitates tethering and the subsequent fusion events. Our model emphasizing the role of Vps1 on late endosome-to-vacuole traffic does not necessarily contradict previous findings that in cells devoid of Vps1 the mistargeted Golgi resident proteins, Kex2 and Vps10, to the plasma membrane are transported to the vacuole by passing through late endosome. It is because of the ability of cells to undergo the delivery of the

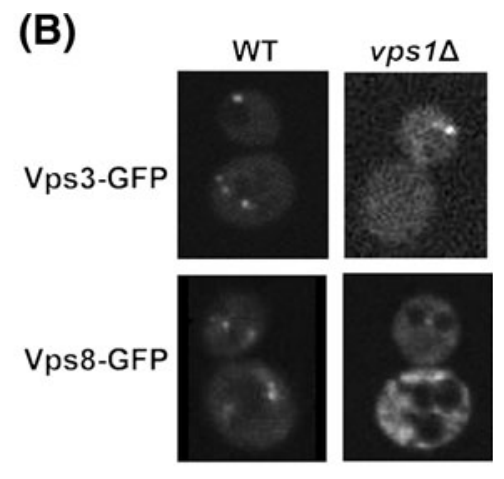

Figure 5. Altered localization of subunits of CORVET and HOPS tethering complexes in vps $1 \Delta$ mutant cells. The indicated genes were tagged with GFP sequences as described in the Material and methods section. (A) Localization of GFP-tagged Vps39 and Vps41, subunits of the HOPS complex in the indicated strains. We found no defect in Vps41-GFP localization in both WT and $v p s 1 \Delta$ cells, whereas the majority ( $~ 80 \%)$ of $v p s 1 \Delta$ cells exhibited no clear vacuolar labelling of Vps39-GFP. (B) Localization of Vps3- and Vps8-GFP. Both GFPtagged subunits of CORVET complex are mislocalized to the cytoplasm in $v p s 1 \Delta$ mutant cells. $61 \%$ and $78 \%$ of Vps3-GFP and Vps8-GFP expressing $v$ ps $1 \Delta$ cells, respectively, exhibited a diffuse cytoplasmic fluorescence. 


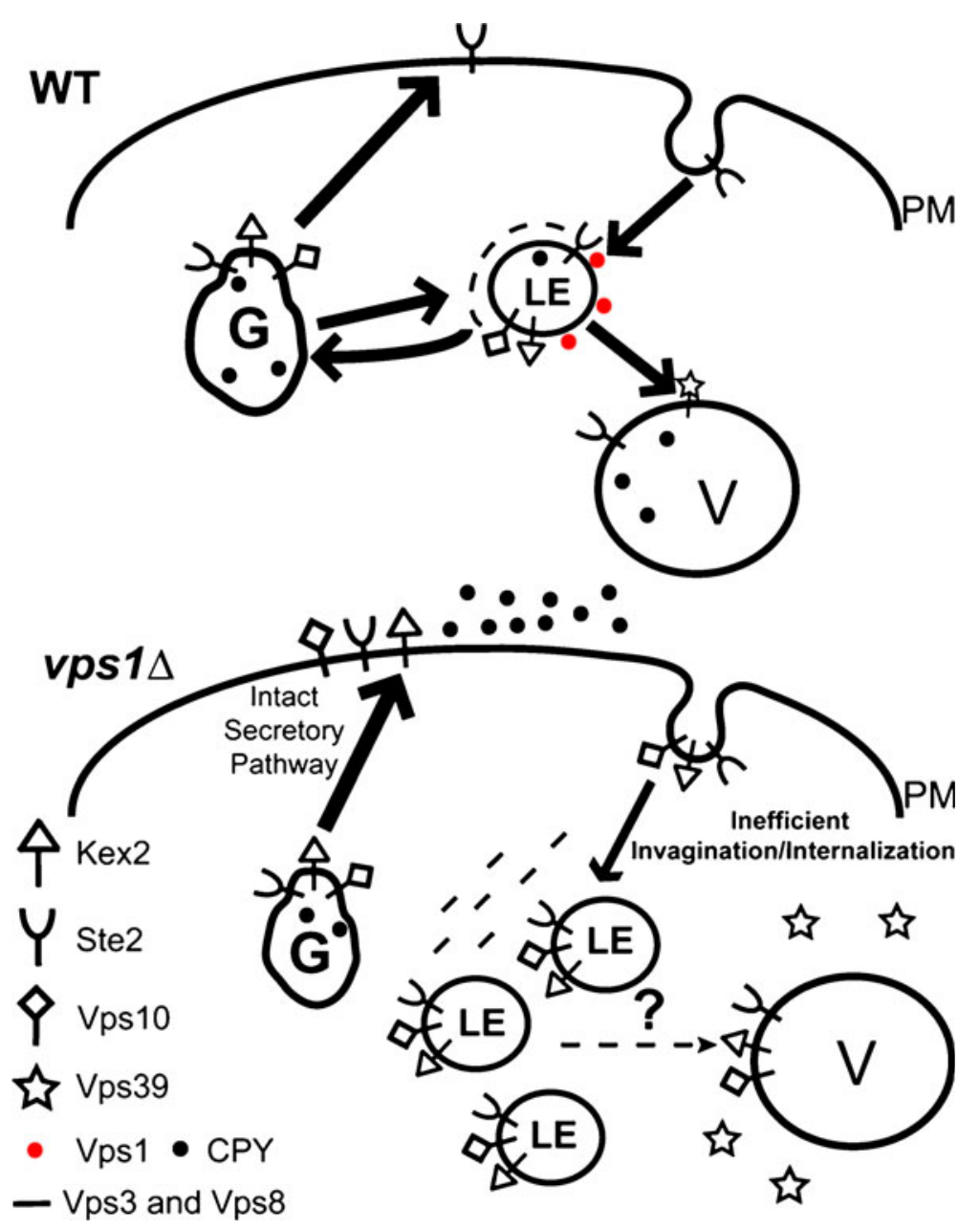

Figure 6. Model of Vps1 function in the transport from late endosome to the vacuole. Golgi body and vacuole are represented by the letters $\mathrm{G}$ and $\mathrm{V}$, respectively, whereas the plasma membrane and late endosomes by PM and LE, respectively. The thick arrows represent pathways that occur properly, whereas the thin and dashed arrows represent pathways that do not occur in an efficient manner. In WT cells, Vps1 does perform multi-functions: (1) for carboxypeptidase Y (CPY) traffic from the late Golgi to late endosomes, (2) for the retrieval process of Vps10 and Kex2, (3) for efficient endocytosis and (4) the last endocytic traffic from late endosome to the vacuole. As shown, in WT cells, only the $\alpha$-factor receptor Ste 2 is endocytosed to be transported to the vacuole, by passing late endosomal compartments that are associated with Vps1. In the absence of Vps1 (B), CPY is secreted out and the two Golgi resident proteins, Kex2 and Vps10, are diverted to PM and then delivered to the late endosome and to the vacuole (Nothwehr et al. 1995). However, endocytic degradation traffics, late endosome-to-vacuole traffic, are significantly impaired in vps $1 \Delta$ cells, resulting in accumulation of late endosomal compartments. The phenotype could be caused by mislocalization of Vps39, a subunit of HOPS complex in the mutant. Additionally, our model suggests the disruption of CORVET complex (Vps3 and Vps8) at the late endosome in the mutant.

Golgi protein toward the vacuole via endocytic traffic, albeit very inefficiently, in the absence of Vps1. Vps1's subcellular localization studies, including ours here, found that Vps1 is present and function at multiple locations such as endocytic sites (Smaczynska-de et al. 2010; Yu and Cai 2004), late endosome, vacuole (Peters et al. 2004) as well as peroxisome (Hoepfner et al. 2001; Vizeacoumar et al. 2006). Unlike mammalian dynamin, Vps1 lacks a pleckstrin homology domain (PHD) that binds phosphatidylinositol lipids within biological membrane (Wang and Shaw 1995). Nevertheless, it was found that Vpslis able to interact and tubulate liposomes in vitro (Smaczynska-de et al. 2010), supporting its appropriate recruitment and in vivo function of Vps1, namely membrane scission. Beside its membrane binding ability, Vps1 can be bound by Pex19, a peroxin involved in peroxisome partitioning (Otzen et al. 2012), for its physical association with peroxisomal membrane (Vizeacoumar et al. 2006). For its vacuolar targeting and function, Vps1 was found to physically interact with the syntaxin-like vacuolar t-SNARE, Vam3 (Peters et al. 2004). Likewise, it is likely that a single or multiple protein factors on endosomal membrane might be involved in the 
recruitment of Vps1, and this aspect remains to be explored. Due to the fact that self-assembly of Vpslis required for liposome tubulation in vitro (Smaczynska-de et al. 2010), we speculate that Vps1, as has been the case for classical dynamins, undergoes self-assembly following its recruitment on endosomal membrane by a yet unknown mechanism. Regardless of its subcellular location, it is thought that Vps1may perform similar function, namely membrane scission. It has been widely accepted that the traffic from late endosome to the vacuole (lysosome) is carried out by a direct fusion between them (Odorizzi et al. 1998; Piper and Luzio 2001), not by vesicular traffic that requires vesicle scission. Prior to the late endosome-vacuole fusion event, a portion of late endosome invaginates and buds into its own lumen to from intralumenal vesicle (ILV), being a part of multivesicular body (MVB). In light of finding that Vps1 may function together with ESCRT complex at late endosome, more specifically, we propose the possibility that $\mathrm{Vps} 1$ plays a role in MVB biogenesis, being a part of the machinery that controls inward budding of ILV. The rationale for the proposed function of Vps1 function is that Vps1 is a functionally conserved dynamin homolog capable of altering membrane morphology. In addition, we favor the proposed Vps1 role, based on the synthetic lethality interaction (SLI) between VPS1 and genes for ESCRT subunits. Nevertheless, it is unclear at the moment how it contributes to the formation of internal vesicle in relation to ESCRT complex.

\section{Acknowledgements}

We are grateful to Nava Segev for providing DsRed-FYVE Plasmid. This work was supported by a National Scientific Foundation Grant 0923024 (to KK) and by thesis funding from Missouri State University (to JH).

\section{References}

Becherer KA, Rieder SE, Emr SD and Jones EW 1996 Novel syntaxin homologue, Pep12p, required for the sorting of lumenal hydrolases to the lysosome-like vacuole in yeast. Mol. Biol. Cell 7 579-594

Brett CL, Plemel RL, Lobingier BT, Vignali M, Fields S and Merz AJ 2008 Efficient termination of vacuolar Rab GTPase signaling requires coordinated action by a GAP and a protein kinase. $J$. Cell Biol. 182 1141-1151

Burd CG and Emr SD 1998 Phosphatidylinositol 3)-phosphate signaling mediated by specific binding to RING FYVE domains. Mol. Cell 2 157-162

Cabrera M, Langemeyer L, Mari M, Rethmeier R, Orban I, Perz A, Brocker C, Griffith J, Klose D, Steinhoff HJ, et al. 2010 Phosphorylation of a membrane curvature-sensing motif switches function of the HOPS subunit Vps41 in membrane tethering. $J$. Cell Biol. 191 845-859
Chang FS, Stefan CJ and Blumer KJ 2003 A WASp homolog powers actin polymerization-dependent motility of endosomes in vivo. Curr. Biol. 13 455-463

Chen DC, Yang BC and Kuo TT 1992 One-step transformation of yeast in stationary phase. Curr. Genet. 21 83-84

Conibear E and Stevens TH 1995 Vacuolar biogenesis in yeast: sorting out the sorting proteins. Cell 83 513-516

Gillooly DJ, Morrow IC, Lindsay M, Gould R, Bryant NJ, Gaullier JM, Parton RG and Stenmark H 2000 Localization of phosphatidylinositol 3-phosphate in yeast and mammalian cells. EMBO J. 19 4577-4588

Hicke L and Riezman H 1996 Ubiquitination of a yeast plasma membrane receptor signals its ligand-stimulated endocytosis. Cell 84 277-287

Hicke L, Zanolari B, Pypaert M, Rohrer J and Riezman H 1997 Transport through the yeast endocytic pathway occurs through morphologically distinct compartments and requires an active secretory pathway and Sec $18 \mathrm{p} / \mathrm{N}$-ethylmaleimide-sensitive fusion protein. Mol. Biol. Cell 8 13-31

Hicke L, Zanolari B and Riezman H 1998 Cytoplasmic tail phosphorylation of the alpha-factor receptor is required for its ubiquitination and internalization. J. Cell Biol. 141 349-358

Hoepfner D, van den Berg M, Philippsen P, Tabak HF and Hettema EH 2001 A role for Vps1p, actin and the Myo2p motor in peroxisome abundance and inheritance in Saccharomyces cerevisiae. J. Cell Biol. 155 979-990

Longtine MS, McKenzie A 3rd, Demarini DJ, Shah NG, Wach A, Brachat A, Philippsen P and Pringle JR 1998 Additional modules for versatile and economical PCR-based gene deletion and modification in Saccharomyces cerevisiae. Yeast 14 953-961

Luo W and Chang A 2000 An endosome-to-plasma membrane pathway involved in trafficking of a mutant plasma membrane ATPase in yeast. Mol. Biol. Cell 11 579-592

Markgraf DF, Ahnert F, Arlt H, Mari M, Peplowska K, Epp N, Griffith J, Reggiori F and Ungermann C 2009 The CORVET subunit Vps8 cooperates with the Rab5 homolog Vps21 to induce clustering of late endosomal compartments. Mol. Biol. Cell 20 5276-5289

Morvan J, Rinaldi B and Friant S 2012 Pkh1/2 dependent phosphorylation of Vps27 regulates ESCRT-I recruitment to endosomes. Mol. Biol. Cell doi:10.1091/mbc.E12-01-0001

Munn AL 2000 The yeast endocytic membrane transport system. Microsc. Res. Tech. 51 547-562

Nannapaneni S, Wang D, Jain S, Schroeder B, Highfill C, Reustle L, Pittsley D, Maysent A, Moulder S, McDowell R, et al. 2010 The yeast dynamin-like protein Vps1:vps1 mutations perturb the internalization and the motility of endocytic vesicles and endosomes via disorganization of the actin cytoskeleton. Eur. J. Cell Biol. 89 499-508

Nickerson DP, Brett CL and Merz AJ 2009 Vps-C complexes: gatekeepers of endolysosomal traffic. Curr. Opin. Cell Biol. 21 $543-551$

Nothwehr SF, Conibear E and Stevens TH 1995 Golgi and vacuolar membrane proteins reach the vacuole in vps1 mutant yeast cells via the plasma membrane. J. Cell Biol. 129 35-46

Obar RA, Collins CA, Hammarback JA, Shpetner HS and Vallee RB 1990 Molecular cloning of the microtubule-associated 
mechanochemical enzyme dynamin reveals homology with a new family of GTP-binding proteins. Nature 347 256-261

Odorizzi G, Babst M and Emr SD 1998 Fab1p PtdIns (3)P 5-kinase function essential for protein sorting in the multivesicular body. Cell 95 847-858

Ostrowicz CW, Meiringer CT and Ungermann C 2008 Yeast vacuole fusion: a model system for eukaryotic endomembrane dynamics. Autophagy 4 5-19

Otzen M, Rucktaschel R, Thoms S, Emmrich K, Krikken AM, Erdmann R and van der Klei IJ 2012 Pex19p contributes to peroxisome inheritance in the association of peroxisomes to Myo2p. Traffic 13 947-959

Peplowska K, Markgraf DF, Ostrowicz CW, Bange G and Ungermann C 2007 The CORVET tethering complex interacts with the yeast Rab5 homolog Vps21 and is involved in endo-lysosomal biogenesis. Dev. Cell 12 739-750

Peters C, Baars TL, Buhler S and Mayer A 2004 Mutual control of membrane fission and fusion proteins. Cell 119 667-678

Piper RC and Luzio JP 2001 Late endosomes: sorting and partitioning in multivesicular bodies. Traffic 2 612-621

Praefcke GJ and McMahon HT 2004 The dynamin superfamily: universal membrane tubulation and fission molecules? Nat. Rev. Mol. Cell Biol. 5 133-147

Price A, Seals D, Wickner W and Ungermann C 2000 The docking stage of yeast vacuole fusion requires the transfer of proteins from a cis-SNARE complex to a Rab/Ypt protein. J. Cell Biol. 148 1231-1238

Ramachandran R 2011 Vesicle scission: dynamin. Semin. Cell Dev. Biol. 22 10-17

Raymond CK, Howald-Stevenson I, Vater CA and Stevens TH 1992 Morphological classification of the yeast vacuolar protein sorting mutants: evidence for a prevacuolar compartment in class E vps mutants. Mol. Biol. Cell 3 1389-1402

Rothman JH and Stevens TH 1986 Protein sorting in yeast: mutants defective in vacuole biogenesis mislocalize vacuolar proteins into the late secretory pathway. Cell 47 1041-1051

Schandel KA and Jenness DD 1994 Direct evidence for ligandinduced internalization of the yeast alpha-factor pheromone receptor. Mol. Cell Biol. 14 7245-7255

Singer-Kruger B, Frank R, Crausaz F and Riezman H 1993 Partial purification and characterization of early and late endosomes from yeast. Identification of four novel proteins. J. Biol. Chem. 268 14376-14386

Singer B and Riezman H 1990 Detection of an intermediate compartment involved in transport of alpha-factor from the plasma membrane to the vacuole in yeast. J. Cell Biol. 110 1911-1922

Smaczynska-de R II, Allwood EG, Aghamohammadzadeh S, Hettema EH, Goldberg MW and Ayscough KR 2010 A role for the dynamin-like protein Vps1 during endocytosis in yeast. J. Cell Sci. 123 3496-3506

Smaczynska-de R II, Allwood EG, Mishra R, Booth WI, Aghamohammadzadeh S, Goldberg MW and Ayscough KR 2012 Yeast dynamin Vps1 and amphiphysin Rvs167 function together during endocytosis. Traffic 13 317-328

Spang A 2009 On the fate of early endosomes. Biol. Chem. 390 753-759

Stefan CJ and Blumer KJ 1999 A syntaxin homolog encoded by VAM3 mediates down-regulation of a yeast $G$ protein-coupled receptor. J. Biol. Chem. 274 1835-1841

Stenmark H and Aasland R 1999 FYVE-finger proteins-effectors of an inositol lipid. J. Cell Sci. 112 4175-4183

Tucker CL and Fields S 2003 Lethal combinations. Nat. Genet. 35 204-205

Vater CA, Raymond CK, Ekena K, Howald-Stevenson I and Stevens TH 1992 The VPS1 protein, a homolog of dynamin required for vacuolar protein sorting in Saccharomyces cerevisiae, is a GTPase with two functionally separable domains. The J. Cell Biol. 119 773-786

Vida TA and Emr SD 1995 A new vital stain for visualizing vacuolar membrane dynamics and endocytosis in yeast. $J$. Cell Biol. 128 779-792

Vizeacoumar FJ, Vreden WN, Fagarasanu M, Eitzen GA, Aitchison JD and Rachubinski RA 2006 The dynamin-like protein Vps1p of the yeast Saccharomyces cerevisiae associates with peroxisomes in a Pex19p-dependent manner. J. Biol. Chem. 281 12817-12823

Wang DS and Shaw G 1995 The association of the C-terminal region of beta I sigma II spectrin to brain membranes is mediated by a $\mathrm{PH}$ domain, does not require membrane proteins and coincides with a inositol-145 triphosphate binding site. Biochem. Biophys. Res. Commun. 217 608-615

Wiederkehr A, Avaro S, Prescianotto-Baschong C, HaguenauerTsapis R and Riezman H 2000 The F-box protein Rcylp is involved in endocytic membrane traffic and recycling out of an early endosome in Saccharomyces cerevisiae. J. Cell Biol. 149 397-410

Wiejak J and Wyroba E 2002 Dynamin: characteristics, mechanism of action and function. Cell Mol. Biol. Lett. 71073 1080

Wurmser AE, Sato TK and Emr SD 2000 New component of the vacuolar class $\mathrm{C}-\mathrm{Vps}$ complex couples nucleotide exchange on the Ypt7 GTPase to SNARE-dependent docking and fusion. J. Cell Biol. 151 551-562

Yu X and Cai M 2004 The yeast dynamin-related GTPase Vps1p functions in the organization of the actin cytoskeleton via interaction with Sla1p. J. Cell Sci. 117 3839-3853 